\title{
D-BRANES IN GROUP MANIFOLDS
}

\author{
SONIA STANCIU
}

\begin{abstract}
In this paper we re-examine the geometric interpretation of gluing conditions in WZW models and the possible D-brane configurations that they give rise to. We show how the boundary conditions are encoded in the gluing conditions imposed on the chiral currents. We analyse two special classes of gluing conditions: the first, which preserves the affine symmetry of the bulk theory, describes D-branes whose worldvolumes are given by 'twisted' conjugacy classes; the second class describes configurations which include subgroups and cosets.
\end{abstract}

\section{Contents}

1. Introduction 1

Note added in prool 4

$2 . \quad$ Boundary conditions and boundary states in flat space 4

Closed string 5

Open string 6

3. Gluing conditions and boundary states in group manifolds 8

Closed string 9

$\begin{array}{ll}\text { Open string } & 10\end{array}$

4. D-branes on group manifolds in the open string sector 11

5. Type-D gluing conditions 13

\begin{tabular}{lll}
\hline 6. & Type-N gluing conditions & 16
\end{tabular}

\begin{tabular}{ll}
\hline 7. Conclusions and outlook & 19
\end{tabular}

Acknowledgements 21

Appendix: (Twisted) conjugacy classes 21

$\begin{array}{ll}\text { References } & 22\end{array}$

\section{INTRODUCTION}

D-branes in group manifolds have received recently a great deal of interest, as they provide an ideal laboratory for the study of the intricacies related to the description of D-branes in general curved backgrounds. There is already an extensive amount of literature devoted to the analysis of D-branes in exact string backgrounds (see, for instance, [1, 2, 园, 田, 5, 6, 7, 8), where by using the methods of CFT one can

Date: July 24, 2013.

Imperial/TP/98-99/59. 
obtain a microscopic description of various D-brane configurations in terms of conformally invariant boundary states and/or boundary conditions. However the geometric interpretation of these configurations proves to be a rather difficult problem, a fact reflected, in particular, in a number of partially contradicting results which appeared in the past few years (e.g. [3, 7, 8, 9]). This has generated a certain controversy in the literature surrounding the very definition and meaning of the Neumann and Dirichlet boundary conditions in curved backgrounds, particularly in the case of a group manifold.

The aim of these notes is twofold. On the one hand, we describe an improved approach to the geometric interpretation of D-branes configurations in group manifolds obtained via the boundary state approach; in this sense, this work is a direct continuation of [4, 7, 9]. Some of the results which we derive here were already used in [9] to determine the possible D-branes in the type IIB string background on $\mathrm{AdS}_{3} \times S^{3} \times T^{4}$ with an NS B field. Here, however, we describe this method in detail and in as much generality as possible at the moment. On the other hand, in the process of doing this, we hope to clarify some discrepancies that can be found in the literature. In order to do this we find it useful to start by reviewing, in Section 2, the well-understood case of the free bosonic string and then work our way up to the more subtle case of group manifolds. Also, in order to be able to directly compare different results we will work, when necessary, both in the closed and in the open string pictures.

The central question in the geometric interpretation of a D-brane configuration obtained via the boundary state approach is finding the correct generalisation of the Neumann and Dirichlet boundary conditions of the free case. As is well-known, boundary conditions in a free bosonic theory are of the form

$$
\partial X^{\mu}(z)=R^{\mu}{ }_{\nu} \bar{\partial} X^{\nu}(\bar{z})
$$

where the information about the Neumann and Dirichlet directions is encoded in the eigenvalues and eigenvectors of the constant matrix $R$. On the other hand, at the quantum level, the above conditions are to be understood as gluing conditions imposed on the chiral currents $\partial X(z)$ and $\bar{\partial} X(\bar{z})$, which are the free fields in terms of which the corresponding conformal field theory is defined. These conditions thus play a crucial role in controlling the conformal invariance of the resulting D-brane configuration.

This type of conditions can further be applied to the study of boundary states in flat backgrounds, or in Calabi-Yau manifolds [1, 2]. In this latter case it has been shown that the geometry of the corresponding D-branes, in the large volume limit, is encoded in the matrix of boundary conditions. 
If we want to study D-branes in curved backgrounds we are faced with two possible avenues (assuming that we are dealing with backgrounds for which we know both the CFT and the corresponding sigma model). We can try to impose gluing conditions on the chiral fields in terms of which the CFT is defined (and then interpret them geometrically) or, alternatively, we can derive the boundary conditions from the sigma model action (and then impose the requirement of conformal invariance). However, since the basic requirement of the boundary state approach is the conformal invariance ${ }^{\text {I }}$ of the resulting D-brane configuration, it appears natural to generalise the above gluing conditions in such a way that conformal invariance remains under control.

In the particular case of strings moving on a group manifold, which are described by the WZW model, the fields in terms of which the conformal field theory is realised are the affine currents. Therefore the natural nonabelian generalisation of the gluing conditions of the free bosonic string takes the form

$$
J_{a}(z)=R_{a}^{b} \bar{J}_{b}(\bar{z}) .
$$

This approach, which is described in Section 3, does however create a problem when it comes to the geometric interpretation of the solutions to these gluing conditions. The reason is the following. In the flat space case the gluing conditions on the free fields $\partial X^{\mu}$ and $\bar{\partial} X^{\mu}$ are, at the same time, boundary conditions taking values in the tangent space of the target manifold at a given but otherwise arbitrary point. By contrast, the nonabelian gluing conditions on the chiral currents of the WZW theory are not, strictly speaking, boundary conditions; rather, since the currents themselves are Lie algebra-valued objects, they take values in the tangent space to the group manifold at the identity. It therefore follows that in order to interpret geometrically the algebraic gluing conditions imposed on the affine currents we must first of all 'translate' them into boundary conditions. In Section 4 we show how this is achieved. It turns out that, unlike the flat space case, the boundary conditions in a group manifold are point dependent, and this fact is essentially due to the nonabelian nature of the target manifold.

The simplest and, in some sense, the most natural class of gluing conditions is the one characterised by the fact that it preserves the infinite-dimensional symmetry of the current algebra. In Section 5 we analyse in detail this type of gluing conditions, which we will refer to as type-D. There we show that type-D gluing conditions defined by an

\footnotetext{
${ }^{1}$ This is not the only condition one must impose in order to get obtain a physically acceptable D-brane spectrum, as it was pointed out recently in 10 . Here, however, we will see that at least in the case of group manifolds, already the conditions imposed independent of a detailed knowledge of the spectrum of the quantum theory restrict significantly the possible D-brane configurations (that is, their geometry).
} 
inner automorphism describe odd-dimensional D-branes whose worldvolumes are conjugacy classes translated by a certain group element. In the most general case, the D-branes worldvolumes describe a generalised version of conjugacy classes which we briefly discuss.

In Section 6 we analyse a different yet closely related type of gluing conditions, which we call type-N. They do not preserve the current algebra of the bulk theory and, generically, they give rise to more complicated D-brane configurations for which we lack, at the moment, a full picture. However we are able to discuss in detail a particular example, for which we identify solutions including subgroups, cosets and open submanifolds of the same dimension as the target manifold.

Finally, in the Appendix we collect a few useful facts about (twisted) conjugacy classes.

\section{Note ADDED IN PROOF}

While this paper was in its final stages a paper [11] appeared where the same problem of D-branes in WZW models is analysed. While there is a certain overlap between some of the results, the two papers use significantly different techniques, and the exact relation between them still remains to be clarified.

\section{BOUNDARY CONDITIONS AND BOUNDARY STATES IN FLAT SPACE}

The aim of this section is to review a few well-known facts about D-branes in a flat string background, in order to set the stage for the discussion of the group manifold case later on.

D-branes can be studied in a variety of ways: by using the techniques of perturbative string theory, they can be described either in terms of boundary conditions of open strings or as boundary states in the closed string sector. Boundary conditions were initially derived from the vanishing of the boundary term in the action of the free string (or, more generally, in the case of a flat background). A Neumann boundary condition imposed in a certain direction ensures that there is no flow of momentum through the boundary of the string worldsheet, that is

$$
\left.\partial_{n} X^{\|}\right|_{\partial \Sigma}=0
$$

Here, the subscript $n$ indicates the direction normal to the boundary of the worldsheet $\partial \Sigma$, whereas the superscript $\|$ denotes the direction parallel to the D-brane worldvolume. By contrast, a Dirichlet boundary condition in a given direction constrains the boundary of the string worldsheet to lie within the hyperplane normal to that direction. This implies the following condition

$$
\left.\partial_{t} X^{\perp}\right|_{\partial \Sigma}=0
$$


where the subscript $t$ refers to the direction tangent to the boundary of the string worldsheet and $\perp$ denotes the component of the background field normal to the worldvolume of the D-brane.

For what follows it will prove useful to write down the Neumann and Dirichlet conditions explicitly, both in the closed and in the open string pictures. For this we need the mode expansion of the free bosonic string

$$
X^{\mu}(\sigma, \tau)=X_{L}^{\mu}(\tau+\sigma)+X_{R}^{\mu}(\tau-\sigma),
$$

where

$$
\partial_{+} X_{L}^{\mu}=\sum_{n \in \mathbb{Z}} \alpha_{n}^{\mu} e^{-i n(\tau+\sigma)}, \quad \partial_{-} X_{R}^{\mu}=\sum_{n \in \mathbb{Z}} \tilde{\alpha}_{n}^{\mu} e^{-i n(\tau-\sigma)},
$$

with the standard notation $\partial_{ \pm}=\partial_{\tau} \pm \partial_{\sigma}$. The canonical commutation relations for the modes read

$$
\begin{aligned}
& {\left[\alpha_{n}^{\mu}, \alpha_{m}^{\nu}\right]=n \delta_{m+n, 0} \eta^{\mu \nu}} \\
& {\left[\tilde{\alpha}_{n}^{\mu}, \tilde{\alpha}_{m}^{\nu}\right]=n \delta_{m+n, 0} \eta^{\mu \nu}} \\
& {\left[\alpha_{n}^{\mu}, \tilde{\alpha}_{m}^{\nu}\right]=0 .}
\end{aligned}
$$

Let us now consider the open and the closed string pictures separately.

Closed string. The boundary of the worldsheet in this case is taken to be at $\tau=0$. A Neumann boundary condition in a given direction say, $X^{\mu}$, is therefore given by the condition

$$
\left.\partial_{\tau} X^{\mu}\right|_{\tau=0}=0
$$

By contrast, a Dirichlet boundary condition in the same direction reads

$$
\left.\partial_{\sigma} X^{\mu}\right|_{\tau=0}=0
$$

One can easily work out these conditions in terms of the modes and obtain

$$
\begin{gathered}
\alpha_{n}^{\mu}+\tilde{\alpha}_{-n}^{\mu}=0, \\
\alpha_{n}^{\mu}-\tilde{\alpha}_{-n}^{\mu}=0 .
\end{gathered}
$$

Notice that the above conditions on the modes are not to be taken as operatorial relations on the quantum fields. In fact, one can easily check that they do not yield automorphisms of the oscillator algebra (11). Rather, they are only satisfied on certain boundary states $|B\rangle$, which are characterised by the very property that they satisfy a certain boundary condition, that is

$$
\begin{aligned}
& \left(\alpha_{n}^{\mu}+\tilde{\alpha}_{-n}^{\mu}\right)|B\rangle=0, \\
& \left(\alpha_{n}^{\mu}-\tilde{\alpha}_{-n}^{\mu}\right)|B\rangle=0,
\end{aligned}
$$

in a given direction $X^{\mu}$.

A boundary state $|B\rangle$ is specified by a complete set of boundary conditions, for all the directions of the target manifold. A convenient way of encoding this information is to introduce a set of linear operators 
$\left\{\phi_{n}^{\mu}\right\}$ which annihilate $|B\rangle$; they are given by $\phi_{n}^{\mu}=\alpha_{n}^{\mu} \pm \tilde{\alpha}_{-n}^{\mu}$, depending on whether we have a Neumann or a Dirichlet boundary condition in the direction $X^{\mu}$. Consistency then requires that a boundary state which is annihilated by two operators, say $\phi_{n}^{\mu}$ and $\phi_{m}^{\nu}$, must also be annihilated by their commutator, that is

$$
\left[\phi_{n}^{\mu}, \phi_{m}^{\nu}\right]|B\rangle=0 \text {. }
$$

One can easily check that this is always satisfied, in other words, we do not have any restriction on the choice of Neumann and Dirichlet conditions that we impose.

Since in practice we usually work in light-cone coordinates on the cylinder or on the complex plane, it is useful to write down the Neumann and Dirichlet boundary conditions in all these coordinates. This is a straightforward exercise, and the results are summarised in the left column of Table 1. Notice in particular that if we pass from the cylinder worldsheet to the annulus in the complex plane, by defining $z=e^{i(\tau+\sigma)}$ and $\bar{z}=e^{i(\tau-\sigma)}$, then the boundary of the worldsheet is at $z \bar{z}=1$.

The most important requirement that a boundary state must satisfy is conformal invariance. In the case of a bosonic theory this means that the holomorphic and antiholomorphic sectors satisfy the following condition at the boundary

$$
(\mathrm{T}(z)-\overline{\mathrm{T}}(\bar{z}))|B\rangle=0,
$$

where, in this case, $\mathrm{T}=\partial X \cdot \partial X$. It is easy to see that any boundary state characterised by an arbitrary combination of Neumann and Dirichlet conditions does satisfy the above condition and therefore preserves conformal invariance.

This set-up can be slightly generalised by introducing a matrix $R$ defining a boundary state of the form

$$
\left(\alpha_{n}^{\mu}+R_{\nu}^{\mu} \tilde{\alpha}_{-n}^{\nu}\right)|B\rangle=0 .
$$

In this case the linear operators which annihilate $|B\rangle$ are given by $\phi_{n}^{\mu}=\alpha_{n}^{\mu}+R^{\mu}{ }_{\nu} \tilde{\alpha}_{-n}^{\nu}$, and the corresponding consistency conditions imply that the matrix $R$ must preserve the flat metric of the target space

$$
R^{T} \eta R=\eta
$$

Open string. The boundary of the string worldsheet it is now at $\sigma=$ 0 . The Neumann boundary condition in the direction $X^{\mu}$ is therefore given now by

$$
\left.\partial_{\sigma} X^{\mu}\right|_{\sigma=0}=0
$$

whereas the Dirichlet boundary condition reads

$$
\left.\partial_{\tau} X^{\mu}\right|_{\sigma=0}=0
$$




\begin{tabular}{|c|c|}
\hline CLOSED STRING & OPEN STRING \\
\hline \hline$\left.\partial_{\tau} X^{\mu}\right|_{\tau=0}=0$ & $\left.\partial_{\sigma} X^{\mu}\right|_{\sigma=0}=0$ \\
$\left.\partial_{\sigma} X^{\mu}\right|_{\tau=0}=0$ & $\left.\partial_{\tau} X^{\mu}\right|_{\sigma=0}=0$ \\
\hline$\left(\alpha_{n}^{\mu} \pm \tilde{\alpha}_{-n}^{\mu}\right)|B\rangle=0$ & $\alpha_{n}^{\mu} \mp \tilde{\alpha}_{n}^{\mu}=0$ \\
\hline$\left(\partial_{+} X^{\mu} \pm \partial_{-} X^{\mu}\right)|B\rangle=0$ & $\left.\left(\partial_{+} X^{\mu} \mp \partial_{-} X^{\mu}\right)\right|_{\sigma=0}=0$ \\
\hline$\left(z^{2} \partial X^{\mu} \pm \bar{\partial} X^{\mu}\right)|B\rangle=0$ & $\left.\left(\partial X^{\mu} \mp \bar{\partial} X^{\mu}\right)\right|_{z=\bar{z}}=0$ \\
\hline$\left(\partial X^{\mu} d z \mp \bar{\partial} X^{\mu} d \bar{z}\right)|B\rangle=0$ & $\left.\left(\partial X^{\mu} d z \mp \bar{\partial} X^{\mu} d \bar{z}\right)\right|_{z=\bar{z}}=0$ \\
\hline
\end{tabular}

TABle 1. The Neumann and Dirichlet boundary conditions for free fields.(In each row, the first relation is a Neumann condition, whereas the second one is a Dirichlet condition.)

Once again, we can write these conditions in terms of the modes, thus obtaining

$$
\begin{aligned}
\alpha_{n}^{\mu}-\tilde{\alpha}_{n}^{\mu} & =0, \\
\alpha_{n}^{\mu}+\tilde{\alpha}_{n}^{\mu} & =0 .
\end{aligned}
$$

However in this case, by contrast to the closed string picture, both the Neumann and Dirichlet boundary conditions preserve the algebra of the modes.

For the sake of completeness, one can write the Neumann and Dirichlet boundary conditions in light-cone coordinates or in the complex plane, where the boundary of the worldsheet is this time at $z=\bar{z}$. The results can be found in the right column of Table 1 .

A short glance at the table reveals the basic rule of thumb that any particular boundary condition in the closed string picture differs by a relative minus sign from the corresponding open string condition. Since this may prove rather confusing in practice, it is maybe worth keeping in mind that the Neumann and Dirichlet boundary conditions, written in terms of the invariant geometric objects, $\partial X d z$ and $\bar{\partial} X d \bar{z}$, have the same form in the closed and open string cases.

Also in this case we can define generalised boundary conditions for the open string by introducing a matrix $R$ satisfying

$$
\left.\left(\partial X^{\mu}-R_{\nu}^{\mu} \bar{\partial} X^{\nu}\right)\right|_{z=\bar{z}}=0 .
$$

The requirement of conformal invariance implies that the matrix $R$ must preserve the metric $\eta$ of the target manifold. Moreover, we have 
the option of imposing that the free field algebra be preserved; however in this case we do not obtain any additional condition on $R$.

\section{GLUing CONDITIONS AND BOUNDARY STATES IN GROUP MANIFOLDS}

Strings moving on a group manifold are described using the WZW model 12, 13, which is a solvable theory, much in the same way as a free theory is. The data necessary to describe such an exact string background is a Lie group $\mathbf{G}$ together with an bi-invariant metric, in terms of which the corresponding WZW action is given by

$$
I[g]=\int_{\Sigma}\left\langle g^{-1} \partial g, g^{-1} \bar{\partial} g\right\rangle+\frac{1}{6} \int_{B}\left\langle g^{-1} d g,\left[g^{-1} d g, g^{-1} d g\right]\right\rangle,
$$

where the field $g$ is a map from a closed orientable Riemann surface $\Sigma$ to the group $\mathbf{G}$. We denote by $\mathfrak{g}$ the corresponding Lie algebra, and we choose for it a basis of generators $\left\{T_{a}\right\}$. The invariant metric on $\mathfrak{g}$ has components $G_{a b} \equiv\left\langle T_{a}, T_{b}\right\rangle$. The exact conformal invariance of this model is based, as is well known, on its infinite-dimensional symmetry group $\mathbf{G}(z) \times \mathbf{G}(\bar{z})$ characterised by the conserved currents

$$
J(z)=-\partial g g^{-1}, \quad \bar{J}(\bar{z})=g^{-1} \bar{\partial} g,
$$

which are the natural dynamical variable in this case. If we introduce the mode expansions for the two currents

$$
J_{a}(z)=\sum_{n \in \mathbb{Z}} J_{a n} z^{-n-1}, \quad \bar{J}_{a}(\bar{z})=\sum_{n \in \mathbb{Z}} \bar{J}_{a n} \bar{z}^{-n-1},
$$

we can write down the affine Lie algebra that they satisfy

$$
\begin{aligned}
& {\left[J_{a n}, J_{b m}\right]=f_{a b}^{c} J_{c n+m}+n \delta_{n+m, 0} G_{a b},} \\
& {\left[\bar{J}_{a n}, \bar{J}_{b m}\right]=f_{a b}^{c} \bar{J}_{c n+m}+n \delta_{n+m, 0} G_{a b},} \\
& {\left[J_{a n}, \bar{J}_{b m}\right]=0 .}
\end{aligned}
$$

It is important to stress that the relative sign in the definition (14) of the two chiral currents is not optional: this definition ensures that the two currents satisfy the same chiral algebra.

The corresponding CFT is then described by the energy-momentum tensor

$$
\mathrm{T}(z)=\Omega^{a b}\left(J_{a} J_{b}\right)(z),
$$

where $\Omega^{a b}$ are components of the inverse of the following invariant metric with the components given by $\Omega=2 G+\kappa$, where $\kappa$ is the Killing form of $\mathfrak{g}$ [14, 15, 16]. The central charge of this CFT is given by $c=\operatorname{dim} \mathfrak{g}-\Omega^{a b} \kappa_{a b}$. In the particular case of a simple Lie group (algebra), the metric $\Omega$ is a multiple of the Killing form, say $\Omega=\mu \kappa$. The usual formulas are then recovered by taking $\mu=\left(x+2 g^{*}\right) / 2 g^{*}$, with $g^{*}$ the dual Coxeter number and $x$ the level. 
So far most attempts to the study of D-branes in group manifolds and coset spaces have tried to exploit the similarities of the WZW model with a free theory (the solvability, the infinite-dimensional symmetry, the exact conformal invariance), in order to construct some kind of nonabelian generalisation of the boundary conditions and boundary states in flat space. In other words, one treats the group manifold case as a generalisation of the flat space case. This means that in the special case of an abelian group one should recover the known results from the flat space case. In particular, the chiral currents of the WZW theory are the nonabelian generalisation of the free bosonic currents, and the affine algebra (5) satisfied by these currents is the natural nonabelian generalisation of the algebra satisfied by the free bosonic fields (1).

In this framework, it appears natural to consider the nonabelian generalisation of the Neumann and Dirichlet conditions written down in the previous section; we will therefore briefly discuss them below. However, we should stress from the very beginning that these gluing conditions for the chiral currents generically are not the same as the Neumann and Dirichlet boundary conditions for a string moving in a group manifold. The detailed derivation of the boundary conditions from the algebraic gluing conditions will follow in the next section. We hope however that the following discussion will shed some light on a few controversial statements in the literature.

Closed string. Since the nonabelian analogues of $\partial X(z)$ and $\bar{\partial} X(\bar{z})$ are $-J_{a}(z)$ and $\bar{J}_{a}(\bar{z})$, respectively, one can immediately generalise the Neumann and Dirichlet conditions for free fields written in terms of the one-forms in the last row in Table 1. One thus obtains

$$
\begin{aligned}
& \left(J_{a}(z) d z+\bar{J}_{a}(\bar{z}) d \bar{z}\right)|B\rangle=0, \\
& \left(J_{a}(z) d z-\bar{J}_{a}(\bar{z}) d \bar{z}\right)|B\rangle=0 .
\end{aligned}
$$

For obvious reasons, as well as for convenience, we will refer to the above conditions as 'Neumann' and 'Dirichlet' gluing conditions, respectively. In terms of the modes, these yield the following

$$
\begin{aligned}
& \left(J_{a n}-\bar{J}_{a-n}\right)|B\rangle=0, \\
& \left(J_{a n}+\bar{J}_{a-n}\right)|B\rangle=0 .
\end{aligned}
$$

Notice that these conditions have an opposite relative sign with respect to the ones written down in [3] (see also [17]). This discrepancy however is not related to the geometric interpretation of these gluing conditions, as implied in [8], but rather lies in the definition of the conserved currents with which one starts $[18$. It must be said that the relative minus sign in the definition of the chiral currents (4) is often neglected in the literature without major consequences; nevertheless, whenever the relation between the holomorphic and the antiholomorphic sectors of the theory comes into play, this sign becomes crucial. 
Alternatively, we can write these gluing conditions on the cylinder where, by using the mode expansion

$$
J_{a}(w)=\sum_{n \in \mathbb{Z}} J_{a n} e^{-n w}, \quad \bar{J}_{a}(\bar{w})=\sum_{n \in \mathbb{Z}} \bar{J}_{a n} e^{-n \bar{w}},
$$

and the fact that the boundary is at $w=-\bar{w}$, we obtain

$$
\begin{aligned}
& \left(J_{a}(w)-\bar{J}_{a}(\bar{w})\right)|B\rangle=0, \\
& \left(J_{a}(w)+\bar{J}_{a}(\bar{w})\right)|B\rangle=0 .
\end{aligned}
$$

The same consistency conditions as in the case of the free theory yield in this case nontrivial restrictions on the type of gluing conditions we can impose in different directions. Indeed, if we impose the same type of gluing conditions in two different directions, say $a$ and $b$, we must then have 'Dirichlet' gluing conditions in the directions $c$ of non-vanishing $f_{a b}{ }^{c}$ since

$$
\left[J_{a n} \mp \bar{J}_{a-n}, J_{b m} \mp \bar{J}_{b-m}\right]|B\rangle=f_{a b}^{c}\left(J_{c n+m}+\bar{J}_{c-(n+m)}\right)|B\rangle .
$$

In particular, we cannot impose 'Neumann' gluing conditions in all directions unless the group is abelian. Moreover, from

$$
\begin{aligned}
{\left[J_{a n}+\bar{J}_{a-n}, J_{b m}-\bar{J}_{b-m}\right]|B\rangle=} & f_{a b}{ }^{c}\left(J_{c n+m}-\bar{J}_{c-(n+m)}\right)|B\rangle \\
& +2 k n \delta_{n+m, 0} G_{a b}|B\rangle,
\end{aligned}
$$

we deduce that we can only have different gluing conditions in orthogonal directions (that is, for which the corresponding element $G^{a b}$ vanishes), and in that case we must have 'Neumann' conditions in the directions $c$ of non-vanishing $f_{a b}{ }^{c}$.

Open string. In this case the nonabelian generalisation of the Neumann and Dirichlet gluing conditions reads

$$
\begin{gathered}
\left.\left(J_{a}(z)+\bar{J}_{a}(\bar{z})\right)\right|_{z=\bar{z}}=0, \\
\left.\left(J_{a}(z)-\bar{J}_{a}(\bar{z})\right)\right|_{z=\bar{z}}=0 .
\end{gathered}
$$

On the modes we obtain

$$
\begin{aligned}
& J_{a n}+\bar{J}_{a n}=0, \\
& J_{a n}-\bar{J}_{a n}=0 .
\end{aligned}
$$

Finally, on the cylinder, where the boundary is at $w=\bar{w}$, we obtain the following gluing conditions

$$
\begin{aligned}
& \left.\left(J_{a}(w)+\bar{J}_{a}(\bar{w})\right)\right|_{w=\bar{w}}=0, \\
& \left.\left(J_{a}(w)-\bar{J}_{a}(\bar{w})\right)\right|_{w=\bar{w}}=0 .
\end{aligned}
$$

We remark that in the open string picture there are no consistency conditions constraining the allowed gluing conditions. If one however is interested in preserving the affine symmetry of the bulk theory then one must keep in mind that, unlike the case of the free open string, here it is only the 'Dirichlet' gluing conditions that preserve the affine 
algebra. In other words, if we want to preserve the current algebra, we cannot impose 'Neumann' gluing conditions in all directions.

This concludes our discussion of the generalisation of the standard Neumann and Dirichlet gluing conditions to the group manifold case. We can now consider the general case, and from now on we will work in the open string picture. Given a string background described by a WZW model with target space $\mathbf{G}$ we consider the following gluing conditions, where from now on we are implicitly evaluating both sides of the condition at the boundary $z=\bar{z}$

$$
J(z)=R \bar{J}(\bar{z})
$$

where $R$ is a linear invertible map $R: \mathfrak{g} \rightarrow \mathfrak{g}$, with $R\left(T_{a}\right)=T_{b} R^{b}{ }_{a}$, for any $T_{a}$ in $\mathfrak{g}$. The basic requirement imposed on $R$ is that the resulting D-brane configurations be conformally invariant. This consistency condition imposes that the holomorphic and antiholomorphic sectors agree at the boundary, that is

$$
\mathrm{T}(z)=\overline{\mathrm{T}}(\bar{z}) .
$$

Using the expression of the energy momentum tensor (6) we obtain that the map $R$ must preserve the metric $\Omega$ :

$$
R^{T} \Omega R=\Omega \text {. }
$$

In the remaining of this paper we will implicitly assume that the matrix of gluing conditions $R$ satisfies this condition.

\section{D-BRANES ON GROUP MANIFOLdS IN THE OPEN STRING SECTOR}

One of the most subtle issues of this approach is extracting the geometric information from a given set of gluing conditions. The precise statement of the problem is the following: given a set of gluing conditions (7) for the chiral currents and a fixed but otherwise arbitrary point $g$ in the target group manifold $\mathbf{G}$, find the possible D-branes which pass through $g$ and are described by these gluing conditions.

It is clearly desirable to interpret the boundary conditions on group manifolds in a similar manner with the flat space case; after all, the special case when $\mathbf{G}$ is abelian is a flat space. However, in spite of the fact that the gluing conditions (7) have been introduced as the nonabelian generalisation of (3), there is an important distinction between them. In the case of a free theory the gluing conditions are formally identical with the boundary conditions defined in the tangent space of the target manifold; therefore the corresponding eigenvalues and eigenvectors of the matrix $R$ identify the Neumann and Dirichlet directions. In the group manifold case the gluing conditions (7) take values in the tangent space of $\mathbf{G}$ at the identity, that is $T_{e} \mathbf{G} \equiv \mathfrak{g}$. This is due to the fact that the currents $J$ and $\bar{J}$ are themselves Lie algebra valued objects. Hence, in order to extract some geometric information out of the algebraic gluing conditions we must first of all 'translate' them into 
boundary conditions in $T_{g} \mathbf{G}$, and then determine what the Neumann and Dirichlet directions are in this case.

Let us consider the left- and right-invariant Maurer-Cartan forms on $\mathbf{G}$, which we denote by $\theta_{L}, \theta_{R}$. We can then use the maps $g$ : $\Sigma \rightarrow \mathbf{G}$ to pull these Maurer-Cartan forms back on $\Sigma$, thus obtaining $g^{*} \theta_{L}=g^{-1} d g$ and $g^{*} \theta_{R}=d g g^{-1}$, which are $\mathfrak{g}$-valued 1 -forms that is, they belong to $\Omega^{1}(\Sigma) \otimes \mathfrak{g}$. We therefore see that the holomorphic current $J(\sigma, \tau)=-\partial g g^{-1}$ takes values in $T^{*} \Sigma^{(1,0)} \otimes \mathfrak{g}$, whereas the antiholomorphic current $\bar{J}(\sigma, \tau)=g^{-1} \bar{\partial} g$ takes values in $T^{*} \Sigma^{(0,1)} \otimes \mathfrak{g}$.

In order to obtain from the Lie algebra valued gluing condition (耳) a boundary condition taking values in $T_{g} \mathbf{G}$, we need to 'translate' the chiral currents from $T^{*} \Sigma \otimes \mathfrak{g}$ to $T^{*} \Sigma \otimes T_{g} \mathbf{G}$. This is done with the help of the two well-known maps, obtained from the left- and right-translation in the group $G$

$$
\begin{aligned}
\left(\lambda_{g}\right)_{*}: \mathfrak{g} \rightarrow T_{g} \mathbf{G}, & \left(\rho_{g}\right)_{*}: \mathfrak{g} \rightarrow T_{g} \mathbf{G}, \\
\left(\lambda_{g}\right)_{*} X=g X, & \left(\rho_{g}\right)_{*} X=X g,
\end{aligned}
$$

which send elements of the Lie algebra into tangent vectors at the point $g$. If we now apply $\left(\rho_{g}\right)_{*}$ to both sides of the gluing condition (7) we obtain the following boundary condition in $T_{g} \mathbf{G}$ :

$$
\partial g=\mathbf{R}(g) \bar{\partial} g
$$

where $\mathbf{R}(g)$ is the map $\mathbf{R}(g): T_{g} \mathbf{G} \rightarrow T_{g} \mathbf{G}$, defined as

$$
\mathbf{R}(g)=-\left(\rho_{g}\right)_{*} \circ R \circ\left(\lambda_{g}\right)_{*}^{-1} .
$$

Thus, for any given tangent vector $V$ in $T_{g} \mathbf{G}$, we have $\mathbf{R}(g)(V)=$ $-R\left(g^{-1} V\right) g$. First of all we must remark that, by contrast with the flat space case, the matrix of boundary conditions $\mathbf{R}(g)$ is not the same as the matrix of gluing conditions $R$, and the former is pointdependent. In general, for an arbitrary group $\mathbf{G}, \mathbf{R}$ only agrees with $R$ at the identity (more generally, for any $g$ in the centre). This implies, in particular, that the identification of the Neumann and Dirichlet boundary conditions used in [7] can only find D-branes which pass through the identity in the group manifold.

We can now safely identify the Neumann and Dirichlet directions. In the same way as in flat space, these are determined by the eigenvalues and eigenvectors of the corresponding matrix of boundary conditions, in our case $\mathbf{R}(g)$. At a given point $g$, a Dirichlet boundary condition corresponds to a -1 eigenvalue of $\mathbf{R}(g)$, which means that the directions normal to the worldvolume of the D-brane are spanned by the corresponding eigenvectors of $\mathbf{R}(g)$. All the other eigenvalues describe Neumann boundary conditions and the corresponding eigenvectors span the tangent space of the worldvolume of the D-brane.

Let us conclude this section by describing an alternative way of deriving the boundary conditions in group manifolds, which proves to be useful in concrete applications. Given a particular group manifold 
G, we can parametrise it by introducing the coordinates $X^{\mu}$, with $\mu=1, \ldots, \operatorname{dim} \mathbf{G}$; we also introduce the left- and right-invariant vielbeins defined by

$$
g^{-1} d g=e_{\mu}^{a} d X^{\mu} T_{a}, \quad d g g^{-1}=\bar{e}_{\mu}^{a} d X^{\mu} T_{a} .
$$

These vielbeins are related by $\bar{e}^{a}{ }_{\mu}=e^{b}{ }_{\mu} A_{b}^{a}$, where $A$ denotes the adjoint action of the group, $g T_{a} g^{-1}=A^{b}{ }_{a} T_{b}$. Using this set-up, one can show that the gluing conditions (7) give rise to the following boundary conditions for the component fields $X^{\mu}$ :

$$
\partial X^{\mu}=\tilde{R}(g)^{\mu}{ }_{\nu} \bar{\partial} X^{\nu} .
$$

Here, the matrix of boundary conditions $\tilde{R}(g)$ is given by

$$
\tilde{R}(g)=-\bar{e}^{-1} R e .
$$

The Neumann and Dirichlet directions are identified in a similar way as before. Moreover, one can see once again that the matrix which describes the boundary conditions at a given point in the target space depends on that point, in this case, through the invariant vielbeins.

Our analysis so far has not made use of any particular property that the map $R$ may have. In the remaining of these notes we will concentrate on two special classes of gluing conditions, for which a detailed analysis of the resulting D-brane configurations is possible.

\section{Type-D GLUing CONDitions}

The defining property of this type of gluing conditions is the fact that the map $R$ is taken to be a Lie algebra automorphism, that is

$$
\left[R\left(T_{a}\right), R\left(T_{b}\right)\right]=R\left(\left[T_{a}, T_{b}\right]\right)
$$

This class can be thought of as a generalisation of D gluing conditions, where the identity is replaced by a Lie algebra automorphism

$$
J_{a}(z)-R_{a}^{b} \bar{J}_{b}(\bar{z})=0 .
$$

They are in some sense the most natural conditions, in that they preserve the infinite-dimensional symmetry of the current algebra. Indeed, from (11) and (8) it follows that $R$ also preserves the metric $G$ and hence the affine algebra (5).

Let us now consider the geometry of the D-brane configurations they describe. If we start with the simplest case, where $R$ is taken to be the identity matrix, then the corresponding matrix of boundary conditions will be given by $\mathbf{R}(g)=-\mathrm{Ad}_{g^{-1}}$, and the resulting $\mathrm{D}$-branes can be identified, as shown in [8], with the conjugacy classes of the group $\mathbf{G}$. Indeed, in this case, provided that the metric $G$ restricts nondegenerately to the conjugacy class $C$ of $g$, the tangent space at $g$ splits into 
the tangent space to the conjugacy class and its perpendicular complement, which can be identified with the tangent space to the centraliser subgroup $Z$ of $g$ :

$$
T_{g} \mathbf{G}=T_{g} C \oplus T_{g} Z \quad \text { with } \quad T_{g} C \perp T_{g} Z .
$$

Moreover $\mathrm{Ad}_{g^{-1}}$ restricts to the identity on $T_{g} Z$, which means that the Dirichlet directions span $T_{g} Z$. Furthermore, the Neumann directions span $T_{g} C$, and hence the worldvolume of the D-brane can be identified with $C$.

As the next step, let us now consider $R$ to be an inner automorphism. It therefore can be identified with $\mathrm{Ad}_{r}$, for some group element $r$, and consequently the gluing conditions (12) become

$$
-\partial g g^{-1}=r g^{-1} \bar{\partial} g r^{-1} \text {. }
$$

If we introduce a new field $\tilde{g}=g r^{-1}$, we can write the corresponding boundary conditions in the following form

$$
\partial \tilde{g}=-\operatorname{Ad}_{\tilde{g}^{-1}} \bar{\partial} \tilde{g} .
$$

By using the previous argument, applied this time to $\tilde{g}$, we are lead to conclude that the corresponding D-brane configuration, which is described by the field $g$, has a worldvolume which lies along the righttranslate $\mathrm{Cr}$ of the conjugacy class of $g$ by the element $r$. This result differs from the one obtained in [8], where it was argued that inner automorphisms, being symmetries of the model, cannot result in Dbrane configurations different from the the ones already described by $R=\mathbb{1}$. Although inner automorphisms are symmetries of the string background, they are not necessarily symmetries of the theory containing a D-brane. This fact does not constitute a novelty, as D-branes break some of the bulk symmetries even in flat space (e.g., translational symmetry).

One remark is in order. One can alternatively apply $\mathrm{Ad}_{r^{-1}}$ in both sides of the gluing condition (13) and obtain, using a similar argument, that the corresponding D-brane lies along the left-translate $r C$ of the conjugacy class of $g$. Since however $C r=r C$, this does not lead to any ambiguity.

It is interesting to point out that conjugacy classes in groups admitting bi-invariant metrics have always even dimension. Indeed, conjugacy classes are the image under the exponential map of adjoint orbits which are diffeomorphic to co-adjoint orbits. Co-adjoint orbits, on the other hand, are symplectic manifolds relative to the natural KirillovKostant-Souriau symplectic structure and hence are even-dimensional. Therefore the D-branes whose worldvolumes can be identified with shifted conjugacy classes have even-dimensional worldvolumes.

We now turn to the case of a general automorphism. We would like to identify the submanifold $N$ of $\mathbf{G}$ that corresponds to the worldvolume of the D-brane described by (12). Provided the metric on $\mathbf{G}$ restricts 
nondegenerately to $N$, we can split the tangent space $T_{g} \mathbf{G}$ into the tangent space to $N$ and its orthogonal complement:

$$
T_{g} \mathbf{G}=T_{g} N \oplus T_{g} N^{\perp} .
$$

Let us consider a given but otherwise arbitrary vector $V$ in $T_{g} N^{\perp}$. As we saw in the previous section, any vector $V$ normal, at a point $g$, to the worldvolume of the D-brane is an eigenvector of the matrix $\mathbf{R}(g)$ corresponding to an eigenvalue equal to -1 . It therefore satisfies

$$
R\left(g^{-1} V\right)=V g^{-1},
$$

which in turn implies that

$$
\left\langle R\left(g^{-1} V\right)-V g^{-1}, R(X)\right\rangle=0,
$$

for any element $X$ in the Lie algebra. From this relation, by using the fact that $R$ preserves the metric, as well as the fact that the metric is bi-invariant, we can deduce that

$$
\langle V, R(X) g-g X\rangle=0 .
$$

This relation tells us that the vector $W_{X} \equiv R(X) g-g X$, which belongs to $T_{g} \mathbf{G}$, is normal to $V$, for any $X$. Hence $W_{X}$ is tangent to $N$ that is, to the worldvolume of the D-brane.

If $R=\mathbb{1}$, then $W_{X}$ would simply be a vector tangent to the conjugacy class $C$ of $g$. In general, $W_{X}$ turns out to be tangent to a 'twisted' conjugacy class, which is defined as follows (for more details, see the Appendix)

$$
C_{R}=\left\{r(h) g h^{-1} \mid h \in \mathbf{G}\right\},
$$

where the map $r: \mathbf{G} \rightarrow \mathbf{G}$ is defined by

$$
r\left(e^{t X}\right)=e^{t R(X)},
$$

for $t$ small enough and $X$ any element in the Lie algebra.

In order to see that $W_{X}$ is tangent to $C_{R}$ we consider a curve in $C_{R}$

$$
\gamma_{R}(t)=r(h(t)) g h^{-1}(t) \text {, }
$$

which is defined in terms of a particular curve $h(t)$ in $\mathbf{G}$, characterised by $h(0)=\mathbb{1}$ and $\dot{h}(0)=X$. This curve passes through $g$ and its tangent vector at $g$ is nothing but $W_{X}$

$$
\left.\frac{d \gamma_{X}(t)}{d t}\right|_{t=0}=R(X) g-g X .
$$

Hence for any Lie algebra element $X$ there exists a tangent vector $W_{X}$ to the D-brane, which is also tangent to the twisted conjugacy class $C_{R}$ of $g$. If we could now show that any tangent vector to the D-brane is of the form $W_{X}$, for some $X$ in $\mathfrak{g}$, then we would be able to conclude that the corresponding D-brane can be identified with $C_{R}$. 
We know that $T_{g} N^{\perp}$ is spanned by the Dirichlet eigenvectors of $\mathbf{R}(g)$. One can rephrase this by saying that any vector $V$ in $T_{g} N^{\perp}$ belongs to the kernel of the following operator

$$
\mathbb{1}-\left(\rho_{g}\right)_{*} \circ R \circ\left(\lambda_{g}\right)_{*}^{-1} .
$$

Therefore $T_{g} N$, which is the orthogonal complement of $T_{g} N^{\perp}$, is nothing but the image of the adjoint of the above operator which is given by

$$
\left(\mathbb{1}-\left(\rho_{g}\right)_{*} \circ R \circ\left(\lambda_{g}\right)_{*}^{-1}\right)^{\dagger}=\mathbb{1}-\left(\lambda_{g}\right)_{*} \circ R \circ\left(\rho_{g}\right)_{*}^{-1} .
$$

In order to obtain the above relation we used the fact that $\left(\rho_{g}\right)_{*} \circ R \circ$ $\left(\lambda_{g}\right)_{*}^{-1}$ is an isometry and therefore its adjoint is given by its inverse. This further implies that any vector $W$ in $T_{g} N$ can be written as

$$
W=U-g R^{-1}\left(U g^{-1}\right),
$$

for some vector $U$ in $T_{g} \mathbf{G}$. Since, moreover, every such vector $U$ in $T_{g} \mathbf{G}$ satisfies $U g^{-1}=R(X)$, for some Lie algebra element $X$, it follows that $W=R(X) g-g X$, for some $X$. We can therefore conclude that the worldvolume of the D-brane can be identified with the twisted conjugacy class $C_{R}$.

Until now we have assumed that the worldvolume of the D-brane forms a submanifold $N$ of $\mathbf{G}$. In order to check that this is indeed the case, one must show that the tangent vectors to $N$ satisfy the Frobenius integrability condition, that is, $\left[W_{1}(g), W_{2}(g)\right]$ belongs to $T_{g} N$ whenever $W_{1}(g)$ and $W_{2}(g)$ do. If we compute this bracket explicitly in our case we obtain

$$
[R(X) g-g X, R(Y) g-g Y]=R([X Y]) g-g[X Y],
$$

where we have used the fact that $R$ is an automorphism. Hence D-type gluing conditions always give rise to D-branes which are submanifolds of $\mathbf{G}$. This implies, in particular, that such gluing conditions cannot describe configurations consisting of intersecting D-branes.

Notice that in this case we do not necessarily obtain odd-dimensional D-branes, as the relation between conjugacy classes and co-adjoint orbits endowed with a symplectic structure cannot be generalised to the 'twisted' case. In fact, in this case, one can find explicit solutions describing even-dimensional D-branes [19].

\section{Type-N GLUing CONDITIONS}

While the particular type of gluing conditions discussed in the previous section is in some sense the most natural, as it preserves the current algebra of the bulk theory, it is certainly not the most general. In this section we consider a different type of gluing conditions which is defined in terms of the Lie algebra automorphism (11). This case, 
however, can be thought of as a generalisation of the 'Neumann' gluing conditions, being defined as follows

$$
J_{a}(z)+R_{a}^{b} \bar{J}_{b}(\bar{z})=0 .
$$

The coefficients $R_{a}^{b}$ are, as before, the matrix elements of the map $R$, with $R\left(T_{a}\right)=T_{b} R_{a}^{b}$, for any $T_{a}$ in $\mathfrak{g}$. Notice that here, although the holomorphic and antiholomorphic sectors are related by a metric preserving automorphism, the infinite-dimensional symmetry of the current algebra is not preserved, contrary to a statement made in [7].

In this case we do not yet understand the general picture of the possible D-brane configurations that one can obtain. For this reason, we will restrict ourselves to considering a concrete example, which is the case of the group $\mathbf{G}=\mathrm{SU}(2)$. It is convenient to choose a parametrisation for $\mathrm{SU}(2)$

$$
g=e^{\theta_{2} T_{2}} e^{\theta_{1} T_{1}} e^{\theta_{3} T_{3}},
$$

where $\theta_{1}, \theta_{2}, \theta_{3}$ play the rôle of the spacetime fields, $\left\{T_{1}, T_{2}, T_{3}\right\}$ forms a basis for $\mathfrak{s u}(2)$, and the brackets are $\left[T_{a} T_{b}\right]=\epsilon_{a b c} T_{c}$. The left- and right-invariant vielbeins read

$e=\left(\begin{array}{ccc}\cos \theta_{3} & \cos \theta_{1} \sin \theta_{3} & 0 \\ -\sin \theta_{3} & \cos \theta_{1} \cos \theta_{3} & 0 \\ 0 & -\sin \theta_{1} & 1\end{array}\right), \quad \bar{e}=\left(\begin{array}{ccc}\cos \theta_{2} & 0 & \cos \theta_{1} \sin \theta_{2} \\ 0 & 1 & -\sin \theta_{1} \\ -\sin \theta_{2} & 0 & \cos \theta_{1} \cos \theta_{2}\end{array}\right)$.

Notice, first of all, that this parametrisation is singular whenever $\cos \theta_{1}=$ 0 . One can in fact show that these 'singular' points give rise to two $S^{1}$ 's inside $S^{3}$, described by

$$
g_{2}\left( \pm \pi / 2, \phi_{2}, \phi_{3}\right)=e^{ \pm \pi / 2 Y_{1}} e^{\left(\phi_{3} \mp \phi_{2}\right) Y_{3}} .
$$

Let us start with the simplest case, $R=\mathbb{1}$, for which the gluing conditions read

$$
J(z)=-\bar{J}(\bar{z}) .
$$

This case can be thought of as the nonabelian generalisation of the flat space case where we have Neumann boundary conditions in all directions. As is well-known, in the flat space case one obtains D-branes whose worldvolume fills the whole target; here the possible D-branes are described by the boundary conditions that (17) give rise to, namely

$$
\partial \theta^{\mu}=\tilde{R}(g)^{\mu}{ }_{\nu} \bar{\partial} \theta^{\nu},
$$

where $\tilde{R}(g)=\bar{e}^{-1} e$. If we evaluate the matrix of boundary conditions at the identity we obtain $\tilde{R}(\mathbb{1})=\mathbb{1}$, which indicates that we have three +1 eigenvalues and hence three Neumann directions spanning $\mathfrak{s u}(2)$. In other words, the identity in $\mathrm{SU}(2)$ belongs to an euclidean D-brane having a three-dimensional worldvolume. As soon as we move away from the identity, $\tilde{R}$ will will no longer be $\mathbb{1}$. In general, $\tilde{R}$ will have 
one +1 and two complex conjugate eigenvalues. Therefore, at generic points in the group manifold, there will be no -1 eigenvalues and thus no Dirichlet directions. Nevertheless, there exist submanifolds of $\mathrm{SU}(2)$ where $\tilde{R}$ has at least one -1 eigenvalue; in fact, since $\operatorname{det} \tilde{R}=1$, it will necessarily have two such eigenvalues. These submanifolds can be described as the zero locus of a function, $F(g) \equiv \operatorname{Tr} \tilde{R}(g)+1$; in our parametrisation this is given by

$$
F=1+\cos \theta_{1} \cos \theta_{2}+\cos \theta_{2} \cos \theta_{3}+\cos \theta_{3} \cos \theta_{1}+\sin \theta_{1} \sin \theta_{2} \sin \theta_{3} .
$$

Let us denote by $\mathcal{F}$ the zero locus of $F$. Since every point in $\mathcal{F}$ is characterised by one +1 and two -1 eigenvalues of $\tilde{R}$, it follows that through every point on $\mathcal{F}$ passes an euclidean D-particle, having as the tangent vector to its worldline the eigenvector of $\tilde{R}$ corresponding to the +1 eigenvalue. Two natural questions arise. First, we would like to have a better geometric understanding of the surface $\mathcal{F}$. Second, we have to determine whether or not these particular solutions are consistent; in other words we must make sure that their worldlines lie on the surface $\mathcal{F}$.

In order to answer the first question we consider the vector fields that generate the adjoint action of a group $\mathbf{G}$. As it is shown in the Appendix, these vector fields can be written in terms of the right- and left-invariant vielbeins as follows

$$
H_{a}(g)=\left(\left(\bar{e}^{-1}\right)_{a}^{\mu}-\left(e^{-1}\right)^{\mu}{ }_{a}\right) \partial_{\mu}, \quad a=1,2,3 .
$$

It is now a straightforward calculation to check that that, in our case, $F$ is annihilated by the corresponding vector fields $H_{a}$

$$
H_{a}(g) \cdot F(g)=0, \quad a=1,2,3 .
$$

This shows that $F$ is a class function on $\mathrm{SU}(2)$, that is $F\left(h g h^{-1}\right)=$ $F(g)$, for any group elements $g$ and $h$. From this we can conclude that the zero locus of $F$ consists of adjoint orbits - that is, conjugacy classes.

Let us now turn to the second question. Since $\tilde{R}$ only takes -1 eigenvalues on the surface $\mathcal{F}$, it means that if we have a solution describing a D-particle its worldline should lie the above surface. In order to check that this is indeed the case we must show that the vector tangent to the worldline is also tangent to $\mathcal{F}$. If we compute explicitly the Neumann eigenvector $V_{1}$ of $\tilde{R}$, we can easily check that

$$
\left.V_{1}(g) \cdot F(g)\right|_{F(g)=0}=0 .
$$

In other words, $V_{1}$ is tangent to the zero locus of $F$, that is $\mathcal{F}$.

Let us summarise our findings so far. We have seen that the gluing conditions (17) give rise two types of euclidean D-branes. Around the identity we have a D2-brane which extends up to a surface defined as 
the zero locus of a class function. Moreover, this surface itself is foliated by the worldlines of D-particles.

Let us now consider the gluing conditions (15) with an arbitrary $R$. Since $\mathfrak{s u}(2)$ is a simple Lie algebra, $R$ is an inner automorphism; hence it can be identified with $\mathrm{Ad}_{r}$, for some group element $r$. By following the same type of argument as in the case of the D-type gluing conditions, one can show that also in this case the effect of an inner automorphism at the level of the gluing conditions is a translation in the group manifold. Indeed, now the matrix of boundary conditions $\tilde{R}=\bar{e}^{-1} R e$ gives rise to three Neumann eigenvectors everywhere except a submanifold which we denote by $\mathcal{F}_{r}$. This submanifold is identified as the zero locus of a function $F_{r}(g)=\operatorname{Tr} \tilde{R}(g)+1$. Clearly, this function is related to the previous $F$, as it satisfies

$$
F_{r}(g)=F\left(g r^{-1}\right) .
$$

Therefore $\mathcal{F}_{r}$ is nothing but the translation of $\mathcal{F}$ by the group element $r$, that is $\mathcal{F}_{r}=\mathcal{F} r$. Hence through every point in $\mathcal{F} r$ passes a D-particle whose worldline lies on $\mathcal{F} r$. Moreover, not only the surfaces $\mathcal{F}$ and $\mathcal{F}_{r}$ are related by translation, but so are the Neumann eigenvectors tangent to the worldlines of the corresponding D-particles on these surfaces.

A particularly interesting case is the one where $R$ itself has two -1 eigenvalues. In this case, the corresponding surface $\mathcal{F} r$ passes through the identity element in $\mathrm{SU}(2)$; therefore there exists a particular Dparticle whose worldline passes through the identity. Its tangent vector, at the identity, is simply given by $V_{1}=\partial_{\theta_{3}}$, as expected. The worldline of this D-particle is nothing but the subgroup of $\mathrm{SU}(2)$ generated by $T_{3}$. Moreover, the translation of this particular solution gives rise to D-particle configurations whose worldlines are cosets in $\mathrm{SU}(2)$.

\section{Conclusions And outlook}

In this paper we have analysed the geometric interpretation of Dbranes in group manifolds obtained via the boundary state formalism. Although the algebraic analysis of the possible gluing conditions is quantum, the subsequent analysis of the geometry of the resulting Dbranes is essentially classical. (This situation is somewhat similar to the analysis in [1, 2] of geometry of D-branes in Calabi-Yau manifolds which was carried out in the large volume limit.) The picture that emerges is the following. The algebraic gluing conditions that one usually imposes on the chiral currents of the WZW model are not to be understood as boundary conditions in the target space; however the latter can be derived from the former by an appropriate 'translation' in the group manifold. The boundary conditions that one obtains in this way are point dependent, by contrast to the more familiar case of the flat space. 
Determining the D-brane configurations that the most general gluing conditions give rise to is a relatively difficult task. Here we have analysed in detail the simplest case of gluing conditions, which preserve the affine symmetry of the bulk theory. The so-called type-D gluing conditions give rise to D-branes whose worldvolumes lie on twisted conjugacy classes. These can be understood as a generalisation of the familiar notion of a conjugacy class, which depends on the automorphism defining the gluing conditions. In the particular case of inner automorphisms we obtain D-branes with even-dimensional worldvolumes. We have also considered a second class of gluing conditions, which we called type-N. They give rise to more complicated configurations which, at least in a particular case, include subgroups, cosets and open submanifolds of dimension equal to the dimension of the target space.

The study carried out in this paper was restricted to the case where the gluing conditions imposed on the affine currents are given in terms of a constant map $R$. This implicit restriction has no conceptual basis, it is merely a practical one, dictated by our inability of working at the level of the quantum theory with field dependent quantities. Field dependent gluing conditions do however appear [7] in the study of the boundary conditions coming from the classical sigma model action of the the WZW model. It would therefore be interesting to consider a generalisation of the formalism presented here in this particular direction.

Here we have taken the point of view that the WZW model is a typical example of an exact string background whose CFT is known explicitly (at least the generators of the conformal (super)algebra); therefore the natural approach to the study of the possible D-brane configurations is to impose gluing conditions on the fields in terms of which the CFT is defined, so that the requirement of conformal invariance can be easily implemented. This is however only one side of the story. The WZW model provides also a typical example of a string background admitting a sigma model description. This allows one to undertake a complementary study of the possible D-brane configurations [20, 18]. Furthermore, one must address the question concerning the relation between the two approaches [7]. In this context it is perhaps worth remarking that the results obtained here, in particular the two types of D-brane configurations in the case of $\mathrm{SU}(2)$, bear a certain similarity with the ones obtained in 20] from the analysis of the open string WZW model. Since the two classes of solutions obtained there are related by Poisson-Lie T-duality, it would be interesting to investigate such a possibility in the framework of the boundary state approach. 


\section{ACKNOWLEDGEMENTS}

It is a pleasure to thank JM Figueroa-O'Farrill and AA Tseytlin for many useful discussions and for a critical reading of the manuscript. Some of the motivation for undertaking this work came from discussions with AY Alekseev and V Schomerus to whom I extend in this way my thanks. This work was supported by a PPARC Postdoctoral Fellowship.

\section{Appendix: (TWisted) CONJugaCy Classes}

Let $\mathbf{G}$ be a connected Lie group and $g$ a fixed but otherwise arbitrary element of $\mathbf{G}$. Let $C(g)$ denote the conjugacy class of the element $g$, defined as the subset of $\mathbf{G}$ with the following elements:

$$
C(g):=\left\{h g h^{-1} \mid h \in \mathbf{G}\right\} .
$$

The conjugacy class of an element $g$ is therefore the orbit of that element under the adjoint action of the group: $\mathrm{Ad}_{h}: \mathbf{G} \rightarrow \mathbf{G}$, defined by $\operatorname{Ad}_{h}(g)=h g h^{-1}$. Each conjugacy class is a connected submanifold of G. Since every element $g$ belongs to one and only one conjugacy class, $\mathbf{G}$ is foliated by its conjugacy classes.

We have seen in Section 5 that it is useful to consider a certain generalisation of the notion of a conjugacy class which is defined as follows:

$$
C_{R}(g):=\left\{r(h) g h^{-1} \mid h \in \mathbf{G}\right\},
$$

where the map $r: \mathbf{G} \rightarrow \mathbf{G}$ is defined by

$$
r\left(e^{t X}\right)=e^{t R(X)}
$$

for $t$ small enough and $X$ any element in the Lie algebra. If we assume that $\mathbf{G}$ is connected then $r$ extends to a Lie group automorphism. Moreover $r$ preserves the bi-invariant metric on the Lie group. The twisted conjugacy class of a given element is therefore the orbit of that element under the action of $\operatorname{Ad}_{h}^{R}: \mathbf{G} \rightarrow \mathbf{G}$, defined by $\operatorname{Ad}_{h}^{R}(g)=$ $r(h) g h^{-1}$.

A curve in $C_{R}(g)$ is generically given by $\gamma_{R}(t)=r(h(t)) g h^{-1}(t)$, with $h(t)$ an arbitrary curve in $\mathbf{G}$. Let us now consider a particular type of curve in $C_{R}(g)$, such that $h(0)=\mathbb{1}$, and $\dot{h}(0)=Y$, with $Y=Y^{a} T_{a}$ an element of the corresponding Lie algebra $\mathfrak{g}$. The tangent vector to this curve at the point $g$ will be given by

$$
\left.\frac{d}{d t}\left(r(h(t)) g h^{-1}(t)\right)\right|_{t=0}=R(Y) g-g Y,
$$

which is an element of $T_{g} \mathbf{G}$. This defines a vector field $H^{R}$ given by

$$
H^{R}(g)=R(Y)_{R}(g)-Y_{L}(g),
$$


where we denote by $Y_{L, R}$ the left- and right-invariant vector fields corresponding to $Y$. One can further show that the components of these invariant vector fields, defined by

$$
Y_{L}(g)=Y_{L}^{\mu}(g) \partial_{\mu}, \quad R(Y)_{R}(g)=R(Y)_{R}^{\mu}(g) \partial_{\mu},
$$

are given by

$$
Y_{L}^{\mu}(g)=Y^{a}\left(e^{-1}\right)^{\mu}{ }_{a}, \quad R(Y)_{R}^{\mu}(g)=Y^{a} R_{a}^{b}\left(\bar{e}^{-1}\right)^{\mu}{ }_{b} .
$$

This implies that the vector field $H^{R}(g)$ which is tangent, at every point $g$ to the curve $\gamma_{R}(t)$ in $C_{R}(g)$ is given by

$$
H^{R}(g)=Y^{a}\left(\left(\bar{e}^{-1}\right)^{\mu}{ }_{b} R_{a}^{b}-\left(e^{-1}\right)^{\mu}{ }_{a}\right) \partial_{\mu} .
$$

This allows us to define $\operatorname{dim} \mathfrak{g}$ vector fields $H_{a}^{R}$, corresponding to each of the generators $T_{a}$ of $\mathfrak{g}$ :

$$
H_{a}^{R}(g)=\left(\left(\bar{e}^{-1}\right)^{\mu}{ }_{b} R_{a}^{b}-\left(e^{-1}\right)^{\mu}{ }_{a}\right) \partial_{\mu} .
$$

These vector fields thus generate the 'twisted' adjoint action of the group $\mathbf{G}$.

The particular case of the standard conjugacy class can be easily obtained by setting $R=\mathbb{1}$. The vector fields that generate the adjoint action of $\mathbf{G}$ read

$$
H_{a}(g)=\left(\left(\bar{e}^{-1}\right)^{\mu}{ }_{a}-\left(e^{-1}\right)^{\mu}{ }_{a}\right) \partial_{\mu} .
$$

\section{REFERENCES}

[1] H. Ooguri, Y. Oz, and Z. Yin, "D-branes on Calabi-Yau spaces and their mirrors," Nuc. Phys. B477 (1996) 407-430. hep-th/9606112.

[2] K. Becker, M. Becker, D. R. Morrison, H. Ooguri, Y. Oz, and Z. Yin, "Supersymmetric cycles in exceptional holonomy manifolds and Calabi-Yau 4-folds," Nuc. Phys. B480 (1996) 225. hep-th/9608116.

[3] M. Kato and T. Okada, "D-branes on group manifolds," Nuc. Phys. B499 (1997) 583-595. hep-th/9612148.

[4] S. Stanciu, "D-branes in Kazama-Suzuki models," Nuc. Phys. B526 (1998) 295-310. hep-th/9708166.

[5] A. Recknagel and V. Schomerus, "D-branes in Gepner models." hep-th/9712186.

[6] J. Fuchs and C. Schweigert, "Branes: from free fields to general backgrounds." hep-th/9712257.

[7] S. Stanciu and A. A. Tseytlin, "D-branes in curved spacetime: the Nappi-Witten background," JHEP 06 (1998) 010. hep-th/9805006.

[8] A. Alekseev and V. Schomerus, "D-branes in the WZW model," Phys. Rev. D60 (1999) 061901. hep-th/9812193.

[9] S. Stanciu, "D-branes in an $A d S_{3}$ background." hep-th/9901122.

[10] J. Harvey, S. Kachru, G. Moore, and E. Silverstein, "Tension is dimension." hep-th/9909072.

[11] G. Felder, J. Fröhlich, J. Fuchs, and C. Schweigert, "The geometry of WZW branes." hep-th/9909030.

[12] D. Gepner and E. Witten, "String theory on group manifolds," Nuc. Phys. B278 (1986) 493-549. 
[13] V. Knizhnik and A. Zamolodchikov, "WZNW models," Nuc. Phys. B247 (1984) 83.

[14] N. Mohammedi, "On bosonic and supersymmetric current algebras for nonsemisimple groups," Phys. Lett. B325 (1994) 371-376. hep-th/9312182.

[15] J. M. Figueroa-O'Farrill and S. Stanciu, "Nonsemisimple Sugawara constructions," Phys. Lett. B327 (1994) 40-46. hep-th/9402035.

[16] J. Figueroa-O'Farrill and S. Stanciu, "Nonreductive WZW models and their CFTs," Nuc. Phys. B458 (1996) 137-164. hep-th/9506151.

[17] N. Ishibashi, "The boundary and crosscap states in conformal field theories," Mod. Phys. Lett. A4 (1989) 251.

[18] K. Gawędzki, "Conformal field theory: a case study." hep-th/9904145.

[19] J. Figueroa-O'Farrill and S. Stanciu, "More D-branes in the Nappi-Witten background." hep-th/9909164.

[20] C. Klimčik and P. Severa, "Open strings and D-branes in WZNW models," Nuc. Phys. B488 (1997) 653-676. hep-th/9609112.

Theoretical Physics Group

BLACKETT LABORATORY

IMPERIAL COLLEGE

LONDON SW7 2BZ, UK

E-mail address: s.stanciu@ic.ac.uk 